\title{
Phenylethanoid glycosides as a possible COVID-19 protease inhibitor: a virtual screening approach
}

\author{
Mario Bernardi ${ }^{1,2} \cdot$ Mohammad Reza Ghaani $^{1} \cdot$ Omer Bayazeid ${ }^{2}$ (i)
}

Received: 27 January 2021 / Accepted: 22 October 2021 / Published online: 3 November 2021

(c) The Author(s), under exclusive licence to Springer-Verlag GmbH Germany, part of Springer Nature 2021

\begin{abstract}
From the beginning of pandemic, more than 240 million people have been infected with a death rate higher than $2 \%$. Indeed, the current exit strategy involving the spreading of vaccines must be combined with progress in effective treatment development. This scenario is sadly supported by the vaccine's immune activation time and the inequalities in the global immunization schedule. Bringing the crises under control means providing the world population with accessible and impactful new therapeutics. We screened a natural product library that contains a unique collection of 2370 natural products into the binding site of the severe acute respiratory syndrome coronavirus 2 (SARS-CoV-2) main protease $\left(\mathrm{M}^{\mathrm{pro}}\right)$. According to the docking score and to the interaction at the active site, three phenylethanoid glycosides (forsythiaside A, isoacteoside, and verbascoside) were selected. In order to provide better insight into the atomistic interaction and test the impact of the three selected compounds at the binding site, we resorted to a half microsecond-long molecular dynamics simulation. As a result, we are showing that forsythiaside $\mathrm{A}$ is the most stable molecule and it is likely to possess the highest inhibitory effect against SARS-CoV-2 $\mathrm{M}^{\text {pro }}$. Phenylethanoid glycosides also have been reported to have both protease and kinase activity. This kinase inhibitory activity is very beneficial in fighting viruses inside the body as kinases are required for viral entry, metabolism, and/or reproduction. The dual activity (kinase/protease) of phenylethanoid glycosides makes them very promising anit-COVID-19 agents.
\end{abstract}

Keywords Natural products $\cdot$ Protease inhibitors $\cdot$ Kinase inhibitors $\cdot$ MMPBSA $\cdot$ Molecular dynamics

$\begin{array}{ll}\text { Abbreviations } \\ \text { Brenk } & \text { Brenk alerts to toxic groups } \\ \text { COVID-19 } & \text { Coronavirus disease } \\ \text { FDA } & \text { US Food and Drug Administration } \\ \text { iLOGP } & \text { Octanol/water partition coefficient } \\ \text { L1 } & \text { Forsythiaside A } \\ \text { L2 } & \text { Isoacteoside } \\ \text { L3 } & \text { Verbascoside } \\ \text { M }^{\text {pro }} & \text { Main protease } \\ \text { MM/PBSA } & \begin{array}{l}\text { Molecular mechanics Poisson-Boltzmann } \\ \text { surface area }\end{array} \\ \text { PL } & \text { Papain-like proteas }\end{array}$

Mohammad Reza Ghaani mohammad.ghaani@ucd.ie

$\triangle$ Omer Bayazeid omerbayazid@gmail.com

1 School of Chemical and Bioprocess Engineering, University College Dublin, Belfield Dublin 4, Ireland

2 Department of Pharmacognosy, Faculty of Pharmacy, Hacettepe University, Ankara 06100, Turkey

$\begin{array}{ll}\text { PGs } & \text { Phenylethanoid glycosides } \\ \text { PME } & \text { Particle-Mesh Ewald } \\ \text { PAINS } & \text { Pan-assay interference compounds } \\ \text { RMSF } & \text { Root mean square fluctuation } \\ \text { RMSD } & \text { Root-mean-square deviation } \\ \text { SARS-CoV-2 } & \begin{array}{l}\text { Severe acute respiratory syndrome corona- } \\ \text { virus 2 }\end{array} \\ \text { WHO } & \text { World Health Organization }\end{array}$

\section{Introduction}

The coronavirus disease (COVID-19) was declared a global pandemic by the World Health Organization (WHO) in March 2020. Of notice, the virus can infect humans and animal causing a wide range of diseases. The current exit strategy involving the spreading of vaccines must be combined with effective treatments. Bringing the crises under control means to provide the world population with accessible and impactful new therapeutics. The WHO solidarity trial of October 2020 reports remdesivir, hydroxychloroquine, 
lopinavir/ritonavir, and interferon to have little to null effect on mortality and duration of the patient's hospitalization. In addition, reinfection cases have been recorded along with the infection of vaccinated people and the common prevention rules were not successful to avoid COVID-19 s wave. Yet vaccines will not be sufficient to save lives on their own that is clearly proved by the multitude of variants spreading around the world. Moderna and Pfizer two-dose vaccines imply the need for a second booster shot, whereas the $\mathbf{J} \& \mathbf{J}$ vaccine takes approximately 29 days to build enough immunity [1]. Because the world-wide distribution is not globally synchronized and equally organized, the virus is expected to be defeated not earlier than 1 year from now at the very least. The lack of "herd immunity" translates in thousands of people risking COVID-19-associated death causes.

Even more importantly, the time has come to face coronaviruses once and for all. Since the 1960s, it is known that humans can be infected by alpha and beta coronaviruses [2]. SARS-CoV, MERS-CoV, and, of course, SARS-CoV-2 all followed the same path involving animal-to-human transition. A virus mutating directly to humans through intermediary species is an event we will not ever be able to get rid of. This underlines the need for therapeutics to indefinitely support our immune system against coronaviruses. In this context, the mechanism of choice for COVID-19 virus degradation is proteolysis. SARS-CoV-2 virus has two proteases: main protease $\left(\mathrm{M}^{\text {pro }}\right)$ and papain-like protease $\left(\mathrm{PL}^{\mathrm{pro}}\right)$. $\mathrm{M}^{\text {pro }}$ plays a crucial role in viral replication which makes it an attractive target for anti-COVID-19 drug design [3].

The main function of protease - also known as proteinase or peptidase - enzyme is to breakdown the peptide bonds of proteins to form smaller polypeptides [4]. Protease enzymes have several functions ranging from food digestion to cancer signaling cascades. In addition, they are also vital for virus's replication and the spread of infectious diseases such as COVID-19 [5]. Based on the protease enzyme active site mechanism, it is possible to list up to 5 different protease groups: aspartyl, cysteine, metalloprotease, serine, and threonine. Their inhibitors can be small molecules or compounds containing one or more peptide chains, and some of the inhibitors can interact with more than a single type of protease [4]. COVID19 main protease is a cysteine protease, characterized by three domains, that catalyzes the breakdown of peptide bonds using histidine (His-41) and cysteine (Cys-145) residues [6]. The catalytic site that is located between domain I and domain II [7]. The main protease of SARSCoV-2 was crystallized with a peptide inhibitor called N3. This peptide forms a covalent bond with the Cys145 and inhibits the protease with half-maximal inhibitory concentration $\left(\mathrm{IC}_{50}\right)$ of $16.77 \mu \mathrm{M}$ [8]. However, the non-covalent bond formed by non-peptide small molecules can have less off-target side effects and toxicity. The most potent $\mathrm{M}^{\text {pro }}$ inhibitor is ML188 (non-covalent inhibitor, $\mathrm{IC}_{50}=12.9 \mu \mathrm{M}$ ) [9]. Due to the reason that there is no specific drugs against SARS-CoV-2, currently there are 16 antiviral drugs that are used against COVID-19, and five of these drugs (lopinavir, ritonavir, darunavir, velpatasvir, and ledipasvi) are HIV and hepatitis C protease inhibitors [10]. On the other hand, Weisberg et al. characterized more than 30 approved kinase inhibitors as potential antiviral agents by inhibiting important kinases required for viral entry, metabolism, and/or reproduction [11] and recently, a combination of baricitinib (kinase inhibitor) and remdesivir (antiviral) has been approved by the US Food and Drug Administration (FDA) for hospitalized adults with COVID-19 [12]. Computer-aided drug design and in silico virtual screening are widely applied in the drug discovery field to rapidly identify a therapeutic solution. Virtual screening approach already proved to be a suitable tool to identify molecules against COVID-19. Mittal et al. identified small molecules (nelfinavir and birinapant) and peptides (pepstatin A and Leupeptin Hemisulphate) as potential inhibitors of SARS-CoV-2 $\mathrm{M}^{\text {pro }}$ [13]. Most of the previous in silico studies have focused mainly on repurposing approved drugs or screening synthetic libraries to target several important SARS-CoV-2 proteins. Our aim is to identify novel natural product scaffolds that can bind to the active site of SARS-CoV-2 $\mathrm{M}^{\text {pro }}$.

In drug discovery, natural products represent a profitable source to find novel small molecules that can act against a series of biological targets. Thus, we screened a natural product library - containing unique collection of 2370 natural products - into the crystal structure of COVID19 main protease. The result of this diversified screening batch highlighted only three potential molecules to target the COVID-19 virus. All these compounds are phenylethanoid glycosides (PGs). PGs are naturally occurring water-soluble small molecules. Their structure is characterized by a phenethyl alcohol (C6-C2) moiety attached to a $\beta$-glucopyranose/ $\beta$-allopyranose through a glycosidic bond. Therefore, PGs are associated to a wide range of biological activities; in fact, kinase inhibitory activity, HIV, and respiratory syncytial virus [14-17] are among the most important targets of PGs. Isoacteoside and verbascoside are renown for potent antiviral activity against respiratory syncytial virus $[14,18]$.

In closing, the research findings presented herein are a combination of drug discovery and a half microsecond-long molecular dynamics simulation. We exploit atomistic simulations as a standard tool to investigate protein-ligand interactions and predict the binding free energy of each indivudal selected compounds with SARS-CoV-2 $\mathrm{M}^{\text {pro }}$. The outcome of our extensive analysis highlights the differences and similarities of three potential PGs natural small molecules that can ultimately act as anti-COVID-19 therapeutics. 


\section{Experimental}

\section{Molecular docking}

The 3D structures of COVID-19 main protease (6LU7) in complex with an inhibitor N3 were downloaded from the Protein Data Bank (www.rcsb.org) [8]. Molecular docking was performed using MOE program (2014.09). Hydrogen atoms and partial charges were added to the protein. Protein minimization was performed with the side chains kept rigid and the ligand flexible. The selected site was isolated and minimized followed by protonating the protein [19]. The natural product library (Catalog No.L1400) which is a collection of unique collection of 2370 natural products was used for docking. The 2D library was convert to a 3D database by energy minimization: forcefield (MMFF94x), gradient $0.001 \mathrm{RMS} \mathrm{kcal} / \mathrm{mol} / \AA \AA^{2}$. The library was docked to the active site of COVID-19 main protease (6LU7.PDB) with scoring affinity London dG and GBVI/WSA dG.

\section{Ligand property analysis}

SwissADME was used to analyze the properties of the three selected compounds. Canonical SMILES of the phenylethanoid glycosides were obtained from PubChem. SMILES were entered into $\mathrm{S}$ wissADME prediction server.

\section{Target identification}

Swiss Target Identification method was used to reveal the possible molecular targets of the three selected compounds [20, 21]. Canonical SMILES of the phenylethanoid glycosides were obtained from PubChem. SMILES were entered into Swiss target prediction server to predict their molecular targets and Homo sapiens was selected as a specie.

\section{Molecular dynamics}

The three selected protein/ligand complexes were subjected to molecular dynamics simulation for $500 \mathrm{~ns}$ using Gromacs 2020.2 [22]. Atomic charges of each ligand were assigned based on the AM1-BCC method of the AmberTools 19 (44) antechamber program. parmchk2 and tleap tools of the AmberTools 19 package were also used to prepare the topology file of the lingads which was converted to gormacs readable format using ACPYPE tool. Gromacs tool (pdb2gmx) was employed to prepare the protein topology parameters based on amber99sb force field. All the complexes were placed in the $96.31 \times 96.31 \times 96.31 \AA$ cubic simulation box and solvated with 28,662 molecules of tip4p water. Counter ions were also added to neutralize the systems. An energy minimisation step was done for each system using asteepest descent integrator for 2000 steps. The NPT ensemble was employed to run the simulations at $300 \mathrm{~K}$ and $1 \mathrm{bar}$ using the Velocity rescaling thermostat (0.1-ps time step) [23] and the Parrinello-Rahman barostat (2.0-ps time constant) [24]. leap-frog integrator was used with a time step of 2 fs [25]. For short range coulombic interactions, a 10.0- $\AA$ cut-off was considered, while long-distance electrostatic interactions were calculated using the Particle-Mesh Ewald (PME) algorithm [26] with a Fourier grid spacing of $1.6 \AA$. Bonds to hydrogen were constrained using the Lincs algorithm [27]. Plumed v2.6.2 [28] and Gromacs 2020.2 [22] and packages were employed for the trajectory analysis. The molecular mechanics Poisson-Boltzmann surface area (MM/ PBSA) method was empolyed to calculate the binding free energies of the protein-ligand complexes using g_mmpbsa software tool $[29,30]$. The overall binding free energy of a protein-ligand complex is defined as follows:

$\Delta \boldsymbol{G}_{\text {binding }}=\Delta \boldsymbol{G}_{\text {elec }}+\Delta \boldsymbol{G}_{\text {polar }}+\Delta \boldsymbol{G}_{\text {vdw }}+\Delta \boldsymbol{G}_{\text {non-polar }}$

where $\Delta G_{\text {elec }}, \Delta G_{\text {polar }}, \Delta G_{\mathrm{vdw}}$, and $\Delta G_{\text {non-polar }}$ are the electrostatic energy, polar solvation energy, van der Waals energy, and nonpolar solvation energy, respectively.

\section{Results and discussion}

PGs are biological active molecules with wide range of activities and they exist in many plant genus such as Veronica, Magnolia, and Forsythia [15]. Out of 2370 natural products, we identify 3 PGs (Fig. 1) with high docking score which interact with amino acids at the binding site, including the catalytic residue His-41. Verbascoside had the highest docking score $(-9.1317, \mathrm{RMSD}=1.51)$ followed by forsythiaside $(-8.0889, \mathrm{RMSD}=2.10)$, and isoacteoside $(-7.7302, \mathrm{RMSD}=1.68)$. The sugar part of forsythiaside A was able to bind to the catalytic residue His-41 and Asp-187 while one aromatic ring formed a hydrogen bond with Thr190 and arene-H bond with Pro-168. Isoacteoside's sugars were able to form many hydrogen bonds at the binding site and one aromatic ring formed a hydrogen bond with Glu166 and the second aromatic ring formed arene-H bond with Gln-189. Verbascoside formed 3 hydrogen bonds with the Thr-24, Asn-142, and Phe-140 and one arene-H bond with Gln-189 (Fig. 2).

To analyze the selected 3 phenylethanoid glycosides' properties, Swiss ADME was used (Table 1) [31-33]. All the three molecules are soluble in water and have low gastrointestinal absorption. We used Swiss model (TargetPrediction) to predict the biological targets of the selected 3 PGs. Swiss model predicted the possible molecular targets along with the actual targets. Targets are ranked according 
<smiles>CC1OC(OCC2OC(OCCc3ccc(O)c(O)c3)C(O)C(O)C2O)C(O)C(O)C1O</smiles>

Forsythiaside A<smiles>CC1OC(OC2C(O)C(COC(=O)/C=C/c3ccc(O)c(O)c3)OC(OCCc3ccc(O)c(O)c3)C2O)C(O)C(O)C1O</smiles>

Isoacteoside<smiles>CC1OC(OC2C(O)C(O)C(O)C(O)C2OC(=O)/C=C/c2ccc(O)c(O)c2)C(O)OC1OCCc1ccc(O)c(O)c1</smiles>

Verbascoside

Fig. 1 Two-dimensional structures of the selected phenylethanoid glycosides

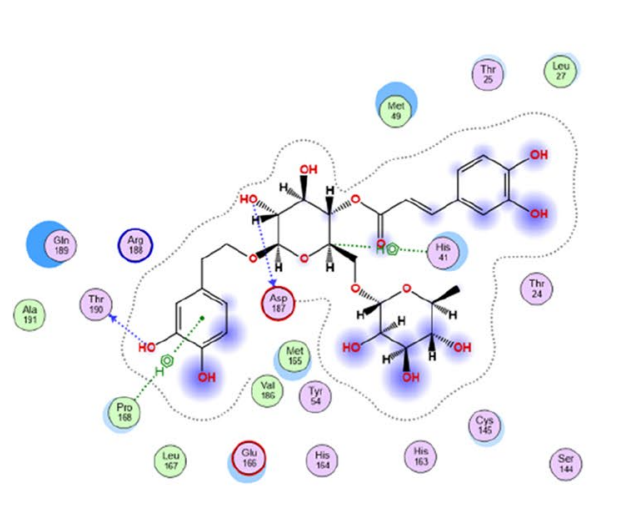

Forsythiaside A

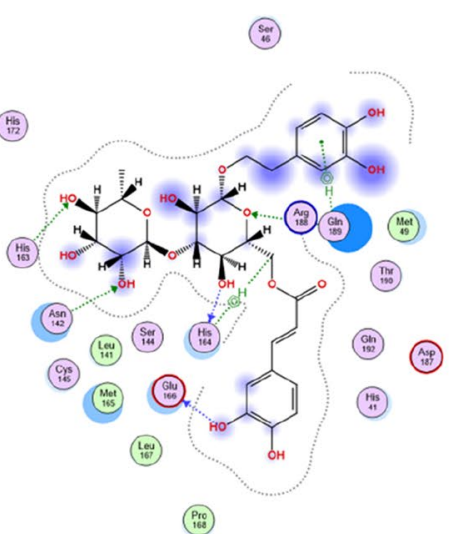

Isoacteoside

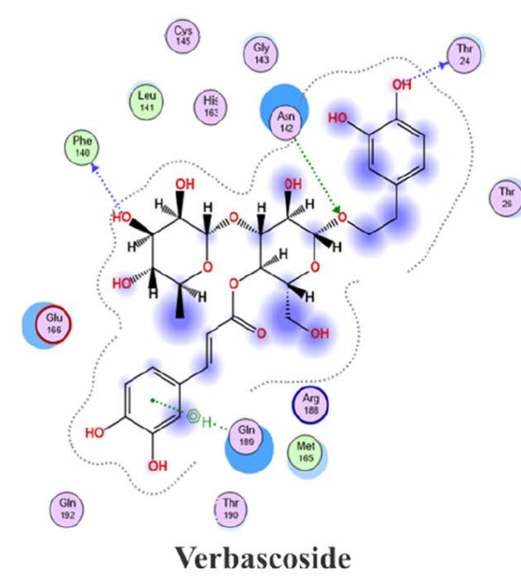

어을 arene-arene

(ㅇ) $\mathrm{H}$ arene-H

(b)+ arene-cation
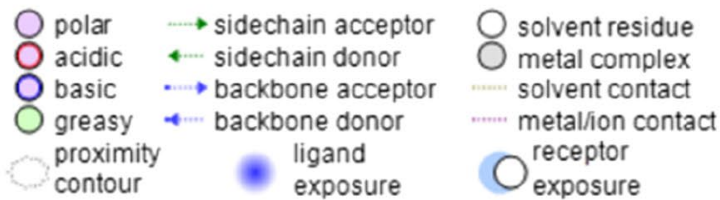

Fig. 2 Two-dimensional ligand interaction in the binding site of COVID-19 main protease (M ${ }^{\text {pro }}$ ) (6LU7.PDB)

to a score that combines both $2 \mathrm{D}$ and $3 \mathrm{D}$ similarity values with the most similar known active molecules to the query molecule [20, 21]. As shown in Fig. 3, PGs exhibit kinase and protease inhibitor activity according to the Swiss model. It has been reported that isoacteoside inhibits the expression of protease protein: matrix metalloproteinase 2, 12, and 13 with $\mathrm{IC}_{50}$ around $10 \mu \mathrm{M}$ [34]. Additionally, forsythiaside and verbascoside inhibit protein kinase $\mathrm{C}$ alpha with $\mathrm{IC}_{50} 1.9 \mu \mathrm{M}$ and $68 \mathrm{nM}$ respectively $[35,36]$.

A recent study reported that protein kinase $\mathrm{C}$ alpha and beta inhibitors have a pro-apoptotic effect in nucleated cells, and this creates a hostile environment for intracellular parasites including viruses. This can enhance the antiviral activity of antivirus drugs [37]. The dual activity (kinase/ protease) of PGs could make them very promising as antiCOVID-19 agents.

From molecular dynamic simulations, we assessed the stability of the protein-ligand bond. A label was associated to each different natural small molecule to improve the readability of the data analysis. Table 2 describes the PG nomenclature adopted herein.

As shown in Fig. 4, the root-mean-square deviation (RMSD) measurement was carried out on each of the three ligands. Interestingly, each ligand reaches a steady configuration at different times within approximately $50 \mathrm{~ns}$. However, L1 stands out for reaching a very steady configuration with respect to the other ligands. This implies a lower noise and mean value of the L1 RMSD. In the 
Table 1 SwissADME results of top three selected compounds with ChemBL IDs

\begin{tabular}{|c|c|c|c|c|c|}
\hline $\begin{array}{l}\text { Compound names } \\
\text { (ChemBL ID) }\end{array}$ & Clinical trial & Lipinsk's rules & Structural alerts & Pharmacokinetics & Water solubility \\
\hline $\begin{array}{l}\text { Forsythiaside A } \\
\text { (CHEMBL504363) }\end{array}$ & - & $\begin{array}{l}3 \text { violations } \\
\mathrm{MW}>500 \\
\mathrm{~N} / \mathrm{O}>10, \mathrm{NH} / \\
\mathrm{OH}>5\end{array}$ & $\begin{array}{l}\text { PAINS }^{1}: 1 \text { alert (catechol A), } \\
\text { Brenk }^{2}: 2 \text { alerts (catechol, } \\
\text { Michael acceptor1) }\end{array}$ & Low GI absorption & Soluble; iLOGP ${ }^{3} 2.15$ \\
\hline $\begin{array}{l}\text { Isoacteoside } \\
\text { (CHEMBL504873) }\end{array}$ & - & $\begin{array}{l}3 \text { violations } \\
\mathrm{MW}>500 \\
\mathrm{~N} / \mathrm{O}>10, \mathrm{NH} / \\
\mathrm{OH}>5\end{array}$ & $\begin{array}{l}\text { PAINS: } 1 \text { alert (catechol A), } \\
\text { Brenk: } 2 \text { alerts (catechol, } \\
\text { Michael acceptor } 1)\end{array}$ & Low GI absorption & Soluble; iLOGP 2.15 \\
\hline $\begin{array}{l}\text { Verbascoside } \\
\text { (CHEMBL444478) }\end{array}$ & Phase II & $\begin{array}{l}3 \text { violations } \\
\mathrm{MW}>500 \\
\mathrm{~N} / \mathrm{O}>10, \mathrm{NH} / \\
\mathrm{OH}>5\end{array}$ & $\begin{array}{l}\text { PAINS: } 1 \text { alert (catechol A), } \\
\text { Brenk: } 2 \text { alerts (catechol, } \\
\text { Michael acceptor } 1)\end{array}$ & Low GI absorption & Soluble; iLOGP 2.15 \\
\hline
\end{tabular}

${ }^{1}$ Pan-assay interference compounds, ${ }^{2}$ Brenk alerts to toxic groups, ${ }^{3}$ Octanol/water partition coefficient

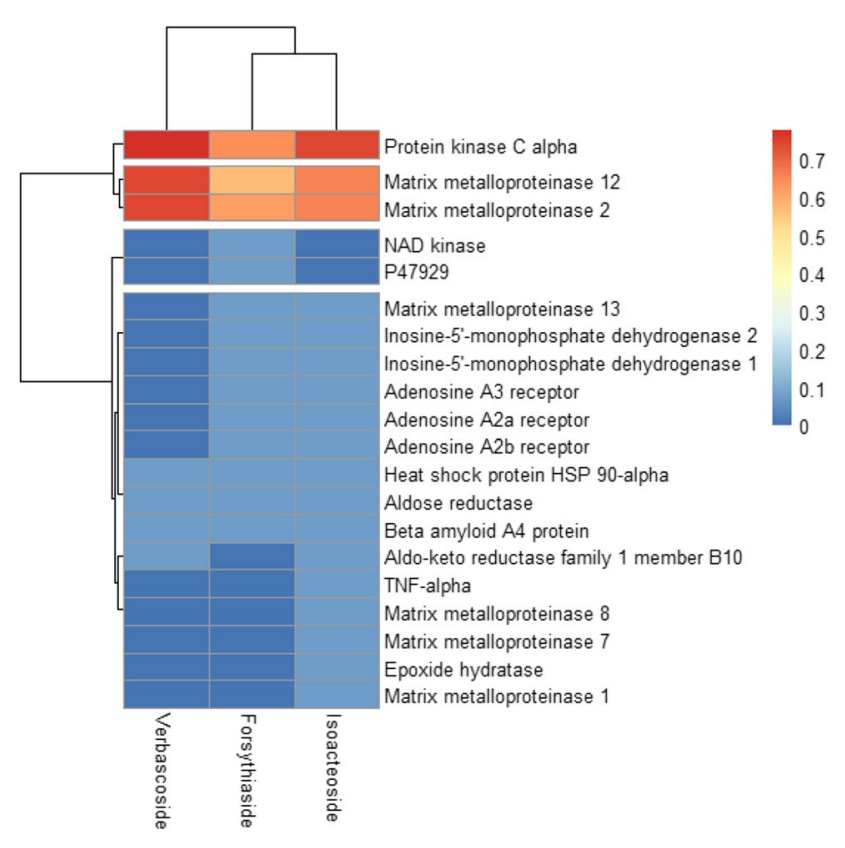

Fig. 3 Consensus molecular targets of the selected 3 phenylethanoid glycosides

Table 2 Compound-associated labels
Supporting information, we are showing that also the protein's RMSD is associated to lower noise when interacting with L1 (Fig. S1).

To further characterize the system, we decided to compute the root mean square fluctuation (RMSF) of atomic positions in the trajectory. In Fig. 5, we consider a subset of residues in the active site corresponding to the "pocket" of interaction. Here, it is evident that different ligands interact in different characteristic ways. There are different moieties of the pocket, highlighted in Fig. 5, where the fluctuation of the atoms depends strongly on the binding ligand. However, the fluctuations in the backbone of the active sites are always limited to less than one Angstrom. Which means that none of the three scenarios implies a detrimental ligand-protease interaction.

The ligand themselves interact with the active site mainly by means of their aromatic moieties. In order to distinguish the two aromatic rings, characteristic of each ligand, we considered their bonding to the central THF heterocyclic ring of Fig. 1. Ring A is defined as the one connected to the central THF heterocyclic ring via a carboxylic group, whereas ring B is not connected via a carboxylic group. Recording the RMSF of the aromatic moieties, reported in Fig. 6, L1turns out to be the most stable compound of the three. In fact, L2 and L3 both show one of the aromatic rings to be fluctuating to twice the extent of the other which allows L2 and L3 to explore different configurations.

This speculation is backed up by the gyration radius data (Fig. S2) of the ligands and the distance between the center of mass of rings A and B. Such a distance, shown in Fig. 7, is indicative of the L1 aromatic rings constraining. Nonetheless, Fig. 7 shows the relative angle between the aromatic rings. This clarifies that $\mathrm{L} 1$ does not explore as many configurations as the other ligands, owing to its 
Fig. 4 RMSD of the ligands during the $0.5-\mu$ s molecular dynamics simulation
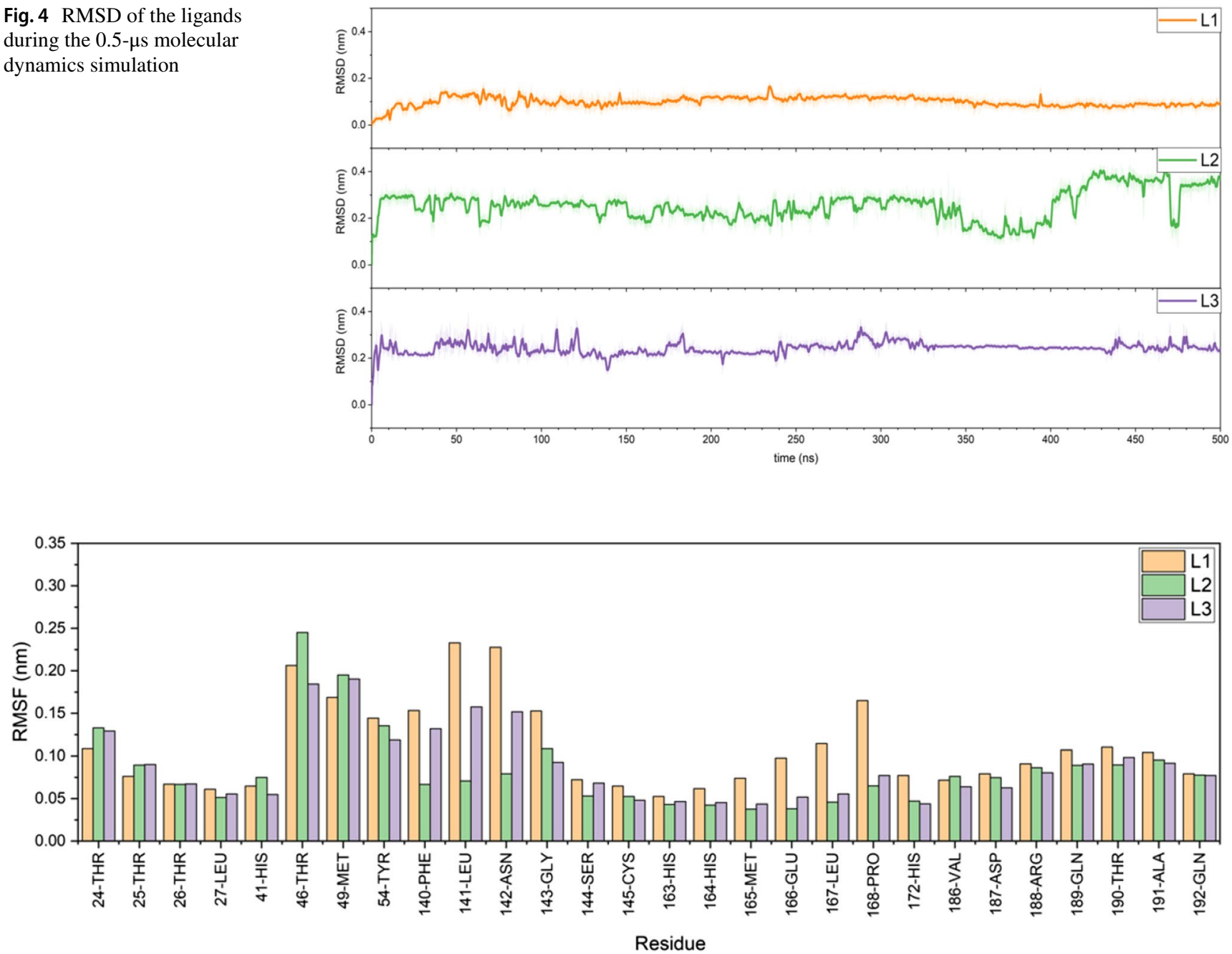

Fig. 5 RMSF of the protein's backbone in pocket site

Fig. 6 RMSF of the characteristic aromatic rings (ring $\mathrm{A}$ and ring B)

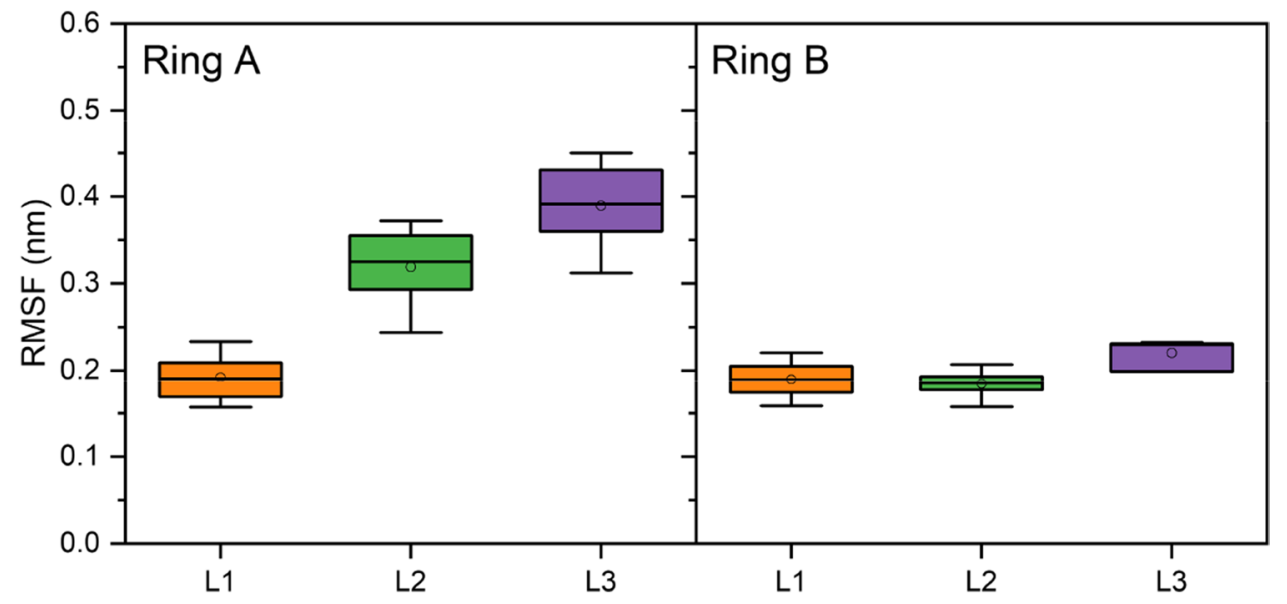

enhanced bond stability. At this point, a mere analysis of the average number of contacts between the ligands and the active site. Figure S3 is not sufficient to determine which structure would be more reliable. Detailed contact map is presented in Fig. S4. Binding free energy 
Fig. 7 Distance and angle between the center of mass of the aromatic rings
Table 3 MMPBSA energy calculated from the last $400 \mathrm{~ns}$ of MD simulation

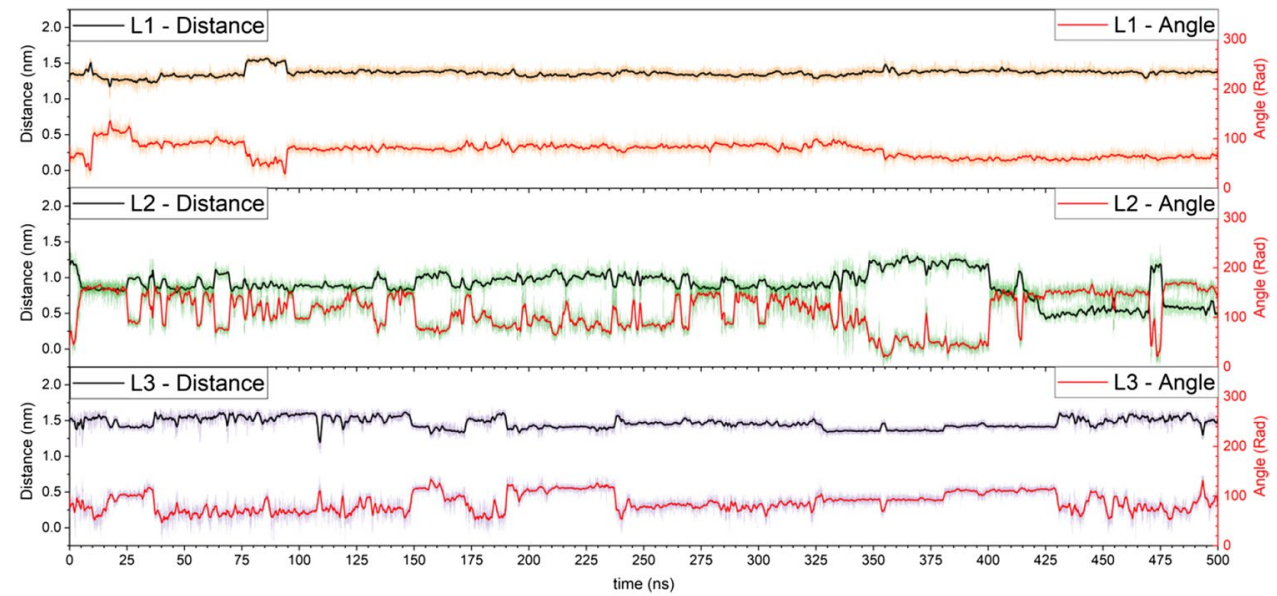

\begin{tabular}{llllll}
\hline $\begin{array}{l}\text { Van der Waal } \\
\text { energy }(\mathrm{kJ} / \mathrm{mol})\end{array}$ & $\begin{array}{l}\text { Electrostatic } \\
\text { energy }(\mathrm{kJ} / \mathrm{mol})\end{array}$ & $\begin{array}{l}\text { Polar solvation } \\
\text { energy }(\mathrm{kJ} / \mathrm{mol})\end{array}$ & $\begin{array}{l}\text { SASA energy } \\
(\mathrm{kJ} / \mathrm{mol})\end{array}$ & $\begin{array}{l}\text { Binding } \\
\text { energy }(\mathrm{kJ} / \\
\mathrm{mol})\end{array}$ \\
\hline L1 & $-253 \pm 24$ & $-64 \pm 25$ & $221 \pm 27$ & $-25 \pm 1$ & $-121 \pm 19$ \\
L2 & $-246 \pm 24$ & $-109 \pm 22$ & $279 \pm 23$ & $-25 \pm 2$ & $-101 \pm 22$ \\
L3 & $-174 \pm 25$ & $-69 \pm 35$ & $211 \pm 45$ & $-21 \pm 2$ & $-53 \pm 18$ \\
\hline
\end{tabular}

calculations allow the measurement of binding strength in protein-ligand or protein-protein complexes [38, 39].

The molecular mechanics of the MM/PBSA method, even though is not as accurate as more computationally intensive methods such as thermodynamic integration [40], has been shown to be satisfactory for the work of both computational and experimental researchers. Last $100 \mathrm{~ns}$ of the MD trajectories was used for the binding free energy calculations for protein/ligand complexes and the result reported in Table 3. According to the result, L1 has a statistically significant lower binding energy in compare with other ligands.

\section{Conclusion}

COVID-19 protease inhibitors should be able to either form hydrogen bonds or trigger a hydrophobic interaction with one of the two catalytic residues: Cys-145 or His-41. Out of 2370 unique natural products library, we identified three PGs as a SARS-CoV-2 $\mathrm{M}^{\text {pro }}$ inhibitors by means of molecular docking. Forsythiaside A was the only molecules among the three PGs to be able to interact with the catalytic residue His-41. Analyzing molecular dynamic trajectories of the selected molecules at the binding site, we identified forsythiaside $\mathrm{A}$ as the most stable one. In fact, this compound is characterized by the steadiest gyration radius and RMSD. The constant relative angle and distance between the aromatic moieties of forsythiaside A represent further evidence of indisputable stability. According to our extensive MD analysis and the resulting binding free energy, this molecule is likely to possess the highest inhibitory effect against SARS-CoV-2 $\mathrm{M}^{\text {pro }}$. Of notice, PGs have been reported to show both protease and kinase activity. Because kinases are required for viral entry, metabolism, and/or reproduction, the inhibitory activity is very beneficial in fighting viruses inside the human body. In fact, the dual activity (kinase/protease) of PGs shall then make them very promising anti-COVID-19 agents.

Supplementary Information The online version contains supplementary material available at https://doi.org/10.1007/s00894-021-04963-2.

Author contribution O.B. conceived the project. O.B. performed the virtual screening, target prediction, and ADME analysis. M.R.G. performed the molecular dynamics. M.B. analyzed the molecular dynamic data. O.B., M.R.G, and M.B wrote and subsequently revised the manuscript.

Availability of data and material N/A.

Code availability N/A.

\section{Declarations}

Ethics approval N/A 
Consent to participate N/A

Consent for publication N/A

Conflict of interest The authors declare no competing interests.

\section{References}

1. Mercado NB et al (2020) Single-shot Ad26 vaccine protects against SARS-CoV-2 in rhesus macaques. Nature 586(7830):583-588

2. Cui J, Li F, Shi Z-L (2019) Origin and evolution of pathogenic coronaviruses. Nat Rev Microbiol 17(3):181-192

3. Xue $X$ et al (2008) Structures of two coronavirus main proteases: implications for substrate binding and antiviral drug design. J Virol 82(5):2515-2527

4. Fear G, Komarnytsky S, Raskin I (2007) Protease inhibitors and their peptidomimetic derivatives as potential drugs. Pharmacol Ther 113(2):354-368

5. Shen H-B, Chou K-C (2009) Identification of proteases and their types. Anal Biochem 385(1):153-160

6. Anand $\mathrm{K}$ et al (2002) Structure of coronavirus main proteinase reveals combination of a chymotrypsin fold with an extra $\alpha$-helical domain. EMBO J 21(13):3213-3224

7. Jin $Z$ et al (2020) Structure of $M$ pro from SARS-CoV-2 and discovery of its inhibitors. 582:289-293

8. Liu X, Wang XJ (2020) Potential inhibitors against 2019-nCoV coronavirus $\mathrm{M}$ protease from clinically approved medicines. $\mathrm{J}$ Genet Genomics 47(2):119-121

9. Augustin TL et al (2020) Novel small-molecule scaffolds as candidates against the SARS coronavirus 2 main protease: a fragmentguided in silico approach. Molecules 25(23):5501

10. Yadav M, Dhagat S, Eswari JS (2020) Emerging strategies on in silico drug development against COVID-19: challenges and opportunities. Eur J Pharm Sci 155:105522

11. Weisberg E et al (2020) Repurposing of kinase inhibitors for treatment of COVID-19. Pharm Res 37(9):167-167

12. Kalil AC et al (2020) Baricitinib plus remdesivir for hospitalized adults with Covid-19. N Engl J Med 384(9):795-807

13. Mittal L et al (2020) Identification of potential molecules against COVID-19 main protease through structure-guided virtual screening approach. J Biomol Struct Dyn 39(10):3662-3680

14. Kernan MR et al (1998) Antiviral phenylpropanoid glycosides from the medicinal plant Markhamia lutea. J Nat Prod 61(5):564-570

15. Xue Z, Yang B (2016) Phenylethanoid glycosides: research advances in their phytochemistry, pharmacological activity and pharmacokinetics. Molecules 21(8):991

16. Kim SN et al (2000) Synthesis and HIV-1 integrase inhibitory activities of caffeoylglucosides. Bioorg Med Chem Lett 10(16):1879-1882

17. Kim KH et al (2015) A new antibacterial octaketide and cytotoxic phenylethanoid glycosides from Pogostemon cablin (Blanco) Benth. Bioorg Med Chem Lett 25(14):2834-2836

18. Chen JL et al (1998) New iridoids from the medicinal plant Barleria prionitis with potent activity against respiratory syncytial virus. J Nat Prod 61(10):1295-1297

19. Bayazeid O, Bedir E, Yalcin FN (2019) Ligand-based virtual screening and molecular docking of two cytotoxic compounds isolated from Papaver lacerum. Phytochem Lett 30:26-30

20. Daina A, Michielin O, Zoete V (2019) SwissTargetPrediction: updated data and new features for efficient prediction of protein targets of small molecules. Nucleic Acids Res 47(W1):W357-W364

21. Gfeller D, Michielin O, Zoete V (2013) Shaping the interaction landscape of bioactive molecules. Bioinformatics 29(23):3073-3079

22. Rakhshani H, Dehghanian E, Rahati A (2019) Enhanced GROMACS: toward a better numerical simulation framework. J Mol Model 25(12):355

23. Bussi G, Donadio D, Parrinello M (2007) Canonical sampling through velocity rescaling. J Chem Phys 126(1):014101

24. Parrinello M, Rahman A (1980) Crystal structure and pair potentials: a molecular-dynamics study. Phys Rev Lett 45(14):1196

25. Parrinello M, Rahman A (1981) Polymorphic transitions in single crystals: a new molecular dynamics method. J Appl Phys 52(12):7182-7190

26. Essmann U et al (1995) A smooth particle mesh Ewald method. J Chem Phys 103(19):8577-8593

27. Hess B et al (1997) LINCS: a linear constraint solver for molecular simulations. J Comput Chem 18(12):1463-1472

28. Bonomi M et al (2019) Promoting transparency and reproducibility in enhanced molecular simulations. Nat Methods 16(8):670-673

29. Kumari R et al (2014) g_mmpbsa-- A GROMACS tool for high-throughput MM-PBSA calculations. J Chem Inf Model 54(7):1951-1962

30. Baker NA et al (2001) Electrostatics of nanosystems: application to microtubules and the ribosome. Proc Natl Acad Sci 98(18):10037-10041

31. Daina A, Michielin O, Zoete V (2017) SwissADME: a free web tool to evaluate pharmacokinetics, drug-likeness and medicinal chemistry friendliness of small molecules. Sci Rep 7:42717

32. Daina A, Michielin O, Zoete V (2014) iLOGP: a simple, robust, and efficient description of $n$-octanol/water partition coefficient for drug design using the GB/SA approach. J Chem Inf Model 54(12):3284-3301

33. Daina A, Zoete V (2016) A boiled-egg to predict gastrointestinal absorption and brain penetration of small molecules. ChemMedChem 11(11):1117

34. Wang L et al (2012) Natural products as a gold mine for selective matrix metalloproteinases inhibitors. Bioorg Med Chem 20(13):4164-4171

35. Zhou B-N et al (1998) Phenylethanoid glycosides from Digitalis purpurea and Penstemon 1 inarioides with $\mathrm{PKC} \alpha$-inhibitory activity. J Nat Prod 61(11):1410-1412

36. Herbert J et al (1991) Verbascoside isolated from Lantana camara, an inhibitor of protein kinase C. J Nat Prod 54(6):1595-1600

37. Ghashghaeinia M et al (2020) Coronavirus disease 2019 (COVID19), human erythrocytes and the PKC-alpha/-beta inhibitor chelerythrine-possible therapeutic implication. Cell Cycle 19(24):3399-3405

38. Gohlke H, Kiel C, Case DA (2003) Insights into protein-protein binding by binding free energy calculation and free energy decomposition for the Ras-Raf and Ras-RalGDS complexes. J Mol Biol 330(4):891-913

39. Hou $\mathrm{T}$ et al (2012) Characterization of domain-peptide interaction interface: prediction of SH3 domain-mediated protein-protein interaction network in yeast by generic structure-based models. J Proteome Res 11(5):2982-2995

40. Wan S et al (2020) Rapid, accurate, precise and reproducible ligand-protein binding free energy prediction. Interface Focus 10(6):20200007

Publisher's note Springer Nature remains neutral with regard to jurisdictional claims in published maps and institutional affiliations. 